\title{
Nanostructured lipid carrier-loaded hyaluronic acid microneedles for controlled dermal delivery of a lipophilic molecule
}

This article was published in the following Dove Press journal:

International Journal of Nanomedicine

31 December 2013

Number of times this article has been viewed

\author{
Sang Gon Lee ${ }^{1, *}$ \\ Jae Han Jeong ${ }^{1, *}$ \\ Kyung Min Lee \\ Kyu Ho Jeong' \\ Huisuk Yang ${ }^{2}$ \\ Miroo $\mathrm{Kim}^{2}$ \\ Hyungil Jung ${ }^{2}$ \\ Sangkil Lee ${ }^{3}$ \\ Young Wook Choi' \\ 'College of Pharmacy, Chung-Ang \\ University, Seoul, South Korea; \\ ${ }^{2}$ Department of Biotechnology, \\ Yonsei University, Seoul, South Korea; \\ ${ }^{3}$ College of Pharmacy, Keimyung \\ University, Daegu, South Korea \\ *These authors contributed equally to \\ this work
}

Correspondence: Young Wook Choi College of Pharmacy, Chung-Ang University, 22I Heuksuk-dong,

Dongjak-gu, Seoul I56-756,

South Korea

Tel +82 28205609

Fax +82 2826 378I

Email ywchoi@cau.ac.kr
Abstract: Nanostructured lipid carriers (NLCs) were employed to formulate a lipophilic drug into hydrophilic polymeric microneedles (MNs). Hyaluronic acid (HA) was selected as a hydrophilic and bioerodible polymer to fabricate MNs, and nile red (NR) was used as a model lipophilic molecule. NR-loaded NLCs were consolidated into the HA-based MNs to prepare NLC-loaded MNs (NLC-MNs). A dispersion of NLCs was prepared by high-pressure homogenization after dissolving NR in Labrafil and mixing with melted Compritol, resulting in $268 \mathrm{~nm}$ NLCs with a polydispersity index of 0.273 . The NLC dispersion showed a controlled release of NR over 24 hours, following Hixson-Crowell's cube root law. After mixing the NLC dispersion with the HA solution, the drawing lithography method was used to fabricate NLCMNs. The length, base diameter, and tip diameter of the NLC-MNs were approximately 350, 380, and $30 \mu \mathrm{m}$, respectively. Fluorescence microscopic imaging of the NLC-MNs helped confirm that the NR-loaded NLCs were distributed evenly throughout the MNs. In a skin permeation study performed using a Franz diffusion cell with minipig dorsal skin, approximately $70 \%$ of NR was localized in the skin after 24-hour application of NLC-MNs. Confocal laser scanning microscopy ( $z$-series) of the skin at different depths showed strong fluorescence intensity in the epidermal layer, which appeared to spread out radially with the passage of time. This study indicated that incorporation of drug-loaded NLCs into MNs could represent a promising strategy for controlled dermal delivery of lipophilic drugs.

Keywords: polymeric microneedles, nanostructured lipid carriers, dermal delivery, controlled release, hyaluronic acid

\section{Introduction}

The stratum corneum (SC) is the outermost layer of the skin. This layer acts as a significant barrier to the penetration of most drugs. ${ }^{1}$ Microneedles (MNs) can penetrate the SC without stimulating nerve fibers in the dermal layer and have been efficiently used to deliver active pharmaceutical ingredients (APIs) into the skin, with negligible pain. $^{2} \mathrm{MN}$ technology has been developed as a means to deliver drugs efficiently to target tissues. Studies conducted using calcein were the first to demonstrate that MNs could increase skin permeability. ${ }^{3}$ Subsequent research has focused on $\mathrm{MN}$ fabrication and their use in drug delivery. MNs vary in length, ranging from $25 \mu \mathrm{m}$ to $1,000 \mu \mathrm{m}$, and can be made from silicon, metal, glass, or polymers. The use of silicon or metal to fabricate MNs carries a risk of brittleness. A very small amount of silicon, metal, or glass deposited in the skin could also elicit inflammatory responses. In contrast, MNs made with biocompatible and biodegradable polymers can be safely applied to the skin..$^{4-6}$ Moreover, polymers are generally cost-effective and suitable 
for mass production. Various polymeric materials such as poly-L-lactic acid, poly-glycolic acid, poly-carbonate, polylactic-co-glycolic acid (PLGA), poly-dimethylsiloxane, a copolymer of methyl vinyl ether and maleic anhydride, carboxymethyl cellulose, maltose, dextrin, and galactose have been used to fabricate MNs. ${ }^{7-11}$

Recently, a PLGA-based MN system was investigated to replace injection using a hypodermic needle. ${ }^{12}$ This minimally invasive system is suitable for self-administration and does not cause pain. However, since PLGA is degraded over a period of months, if it remains stuck to the skin, the recovery of skin barrier function at the injection site may be delayed. To overcome these limitations, hyaluronic acid (HA)-based MNs have been introduced. HA, which is a widely used ingredient in skin care products, is a biocompatible and biodegradable polymer that dissolves rapidly in the biological milieu. ${ }^{13}$ An additional advantage is that fabrication of HA-based MNs does not require the use of organic solvents, because of HA's solubility in water. ${ }^{14}$ However, it is difficult to incorporate many lipophilic APIs, including retinoids, corticosteroids, arildone, resveratrol, and cyclosporine, into these hydrophilic polymeric MNs. Although the research literature on this type of drug loading is very limited, one successful study described encapsulation of a hydrophobic drug molecule into PLGA nanoparticles, which were then dispersed homogenously in water-soluble polymeric MNs. ${ }^{15}$

Maintenance of appropriate drug concentrations in the skin is necessary in many dermatological conditions such as pigmentation, vitiligo, topical infections, and psoriasis. Although MNs greatly enhance drug permeation rates, the permeated drugs are rapidly absorbed into the blood stream, leaving only a small quantity of drugs localized on the skin. ${ }^{16}$ For controlled dermal delivery, incorporation of a particulate drug carrier within MNs has been investigated. ${ }^{12,15,17}$ Recently, a particle-based MN system was introduced to develop a sustained skin immunization-based therapeutic strategy. This system entraps the antigen within a polymeric nanocarrier to prolong antigen release to the skin-resident dendritic cells. ${ }^{17}$ Once the nanocarrier is liberated from the $\mathrm{MN}$ and is exposed to the dermal layer, it can control the release of the entrapped drug. Lipid-based particulate carriers, including solid lipid nanoparticles (SLNs) and nanostructured lipid carriers (NLCs), have been introduced for formulation of lipophilic drugs into water-soluble polymeric matrices, since these drug-loaded nanoparticles can be dispersed homogeneously in an aqueous phase. NLCs represent the next generation of SLNs, consisting of liquid oil and solid lipid.
Lipophilic APIs are easily dissolved or dispersed in the lipid phase, resulting in a high payload capacity. ${ }^{18}$ Because the lipid phase of NLCs is biocompatible, there is no immune rejection. ${ }^{19}$ These formulations are particularly useful for topical application, because their nanosize ensures close contact with the skin cells and helps them to remain on skin tissue as a thin occlusive film, thereby enabling controlled drug release. ${ }^{18}$ Studies of lipid nanoparticles have recently been extended for the development of efficient loading methods for therapeutic proteins, confirmation of structural assembly, and surface modification to target specific sites. ${ }^{20-22}$

In the present study, NLCs were employed to formulate a lipophilic drug into hydrophilic polymeric MNs. HA was selected as a hydrophilic and bioerodible polymer to fabricate the MNs, and nile red (NR) was used as a model probe representing a lipophilic API. NR-loaded NLCs were consolidated into the HA-based MNs to prepare NLCMNs. NR entrapment in NLCs, the physical characteristics of NLC-MNs in terms of homogeneity of NR distribution and $\mathrm{MN}$ array specifications, and the release of NR from either NLCs or NLC-MNs were evaluated. Finally, the skin distribution behavior of NR was examined by both quantitative determination and microscopic image analyses.

\section{Materials and methods Materials}

NR and Tween 80 were purchased from Sigma-Aldrich (St Louis, MO, USA). Compritol ${ }^{\circledR} 888$ ATO (glyceryl behenate) and Labrafil M 1944 CS (oleyl macrogol glycerides) were kindly gifted by Gattefossé (Saint-Priest, France). HA sodium salt (29 kDa) was purchased from Soliance (Pomacle, France). Methanol was purchased from JT Baker (Avantor Performance Materials, Center Valley, PA, USA). Double-distilled water was used for all experiments. Minipig skin was purchased from Medi Kinetics Co., Ltd. (Pyeongtaek, Gyeonggi-do, Korea).

\section{NR assay}

The quantitative determination of NR was performed by highperformance liquid chromatography (HPLC). The system consisted of a pump (L-7100), an autosampler (L-7200), a fluorescence detector (L-7485), and a data station (LaChrom Elite; Hitachi Ltd., Tokyo, Japan). NR was separated using a Capcell pak C18, $0.5 \mu \mathrm{m}, 4.6 \times 150 \mathrm{~mm}$ (Shiseido Company, Limited, Tokyo, Japan) with methanol:water (93:7) as the mobile phase, at a flow rate of $1.0 \mathrm{~mL} /$ minute. The injection volume was $50 \mu \mathrm{L}$ and NR was detected at $599 \mathrm{~nm}$. The intraday and interday precision and accuracy of the NR assay 
were estimated in triplicate at three different concentrations: 10,60 , and $100 \mathrm{ng} / \mathrm{mL}$. The calibration curve was based on seven different standard concentrations. Calibration curves were used to determine the limit of detection and limit of quantitation.

\section{Preparation of NLCs and NLC-MNs}

NLCs were prepared using the high-pressure homogenization (HPH) method. NR (0.5 mg) was dissolved in Labrafil $(0.2 \mathrm{~mL})$ and mixed with melted Compritol $(0.3 \mathrm{~g})$. Nine milliliters of an aqueous phase containing Tween 80 (2.5\%) was heated to $85^{\circ} \mathrm{C}$ and mixed with the lipid phase. The mixture was sonicated for 60 seconds (Model 2210; Branson Ultrasonics Co, Danbury, CT, USA). The hot pre-emulsion was further processed by HPH, applying 10 cycles at 500 bar (M-110S; Microfluidics, Westwood, MA, USA) to obtain an oil/water nanosuspension. This was gradually cooled down to room temperature and stored in a refrigerator.

To fabricate NLC-MNs, the NLC dispersion (10 mL) was mixed with HA sodium salt $(0.2 \mathrm{~g})$. The mixture was dropped onto a $1-\mathrm{cm}^{2}$ carboxymethyl cellulose base plate in a $7 \times 7$ arrangement using solution dispenser (ML-5000X; Musashi Engineering, Inc., Tokyo, Japan) and automated X-, Y-, and Z-stage (SHOT mini 100-s; Musashi Engineering, Inc.). The droplets were then transformed into MNs by the drawing lithography method (Figure 1). ${ }^{23}$ After the pillar contacted the $7 \times 7$ pattern of mixture droplets, drawing resulted in the appearance of a conical bridge between the plate and pillar.
The desired liquid bridge was dried to generate a rigid structure. Isolation drawing was performed to separate the MNs from the pillar.

\section{Characterization of NLCs}

The NLC dispersion was diluted 1,000 times with water, and the particle size and zeta potential were measured using photon correlation spectroscopy (Zetasizer Nano-ZS; Malvern Instruments, Malvern, UK) with a $50 \mathrm{mV}$ laser at a scattering angle of $90^{\circ}$. The drug encapsulation efficiency (EE) $(\%)$ of NR in the NLC dispersion was determined by an ultrafiltration centrifugation method. ${ }^{24}$ The amount of unencapsulated drug was determined indirectly by ultrafiltration using centrifugal filter tubes (Amicon Ultra-4; EMD Merck Millipore, Billerica, MA, USA) with a molecular weight cutoff of $30 \mathrm{kDa}$. The amount of NR encapsulated was calculated by the difference between the total amount used to prepare the systems and the amount of NR that remained in the aqueous phase after isolation of the systems. Free and total NR concentrations were measured using HPLC, and EE (\%) was calculated using the following formula: $[(\mathrm{D}-\mathrm{C}) / \mathrm{D}] \times 100$, where $\mathrm{D}$ is the total amount of drug added and $\mathrm{C}$ is the amount of drug detected in the water phase. ${ }^{25}$

Meanwhile, to determine NR loading into the NLCs, $10 \mu \mathrm{L}$ of NLC dispersion was diluted with methanol and sonicated in a bath-type sonicator at $40^{\circ} \mathrm{C}$ for 10 minutes to dissolve the NR from the lipid carriers. ${ }^{26}$ Drug loading (mg NR/g lipid) was calculated using the following

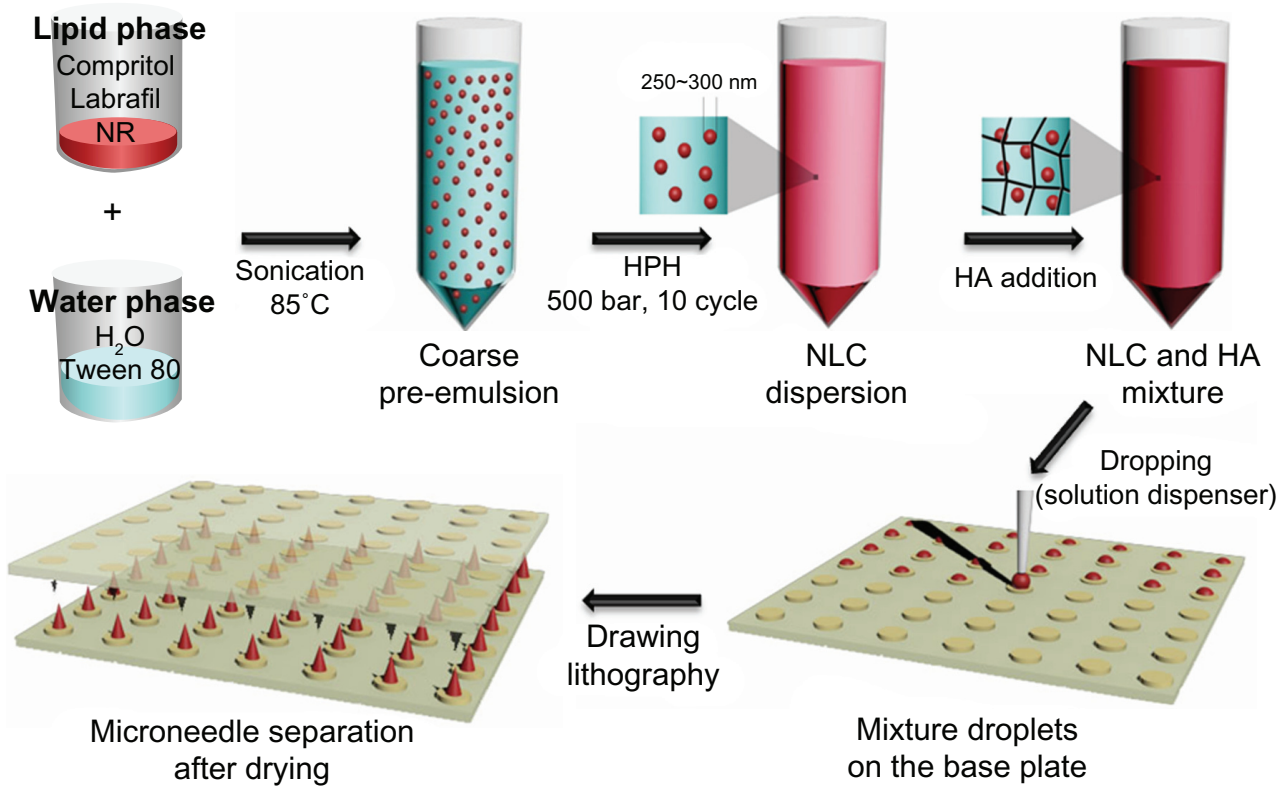

Figure I Schematic illustration for the NLC-MN fabrication procedure.

Abbreviations: HA, hyaluronic acid; HPH, high-pressure homogenization; NLC-MN, nanostructured-lipid-carrier-loaded microneedle; NR, nile red; NLC, nanostructured lipid carrier. 
formula: $(\mathrm{R}-\mathrm{C}) / \mathrm{L}$, where $\mathrm{R}$ is the total amount of $\mathrm{NR}$ remaining in the NLC dispersion, $\mathrm{C}$ is the amount of drug detected in the water phase, and $\mathrm{L}$ is the total amount of lipid added. All measurements were carried out under ambient conditions and in triplicate.

\section{In vitro release of NR}

The release of NR from NLCs was determined in vitro using Franz diffusion cells with donor and receptor compartments, separated by a membrane filter. Cellulose nitrate membrane filters with a pore size of $0.1 \mu \mathrm{m}$ (7181-004; GE Healthcare Europe GmbH, Freiburg, Germany) were mounted in the diffusion cells. The receptor phase was filled with distilled water containing 5\% Tween 80 as an acceptor medium to achieve sink conditions during the experiment, and water-jacketed at $37^{\circ} \mathrm{C}$. NLC dispersion $(100 \mu \mathrm{L})$ was applied to the donor compartment, which had an available diffusion area of $1.76 \mathrm{~cm}^{2}$. Aliquots $(300 \mu \mathrm{L})$ were withdrawn from the receptor compartment $(11 \mathrm{~mL})$ at predetermined time intervals for 48 hours and diluted 2.5 -fold with water. Separately, an NR-containing micellar solution was prepared with Tween $80(5 \% \mathrm{v} / \mathrm{v})$ as a control, and the release of NR from micelles was observed using the procedure described above. After HPLC determination, the cumulative amount of NR in the receptor phase was plotted as a function of time.

\section{Characterization of NLC-MNs}

The length, base diameter, and tip diameter of seven arrays of NLC-MNs were determined in triplicate by microscopic observation (Motic AE31; Speed Fair Co, Ltd, Hong Kong). The aspect ratio was calculated by the ratio of length to base diameter. The amount of NR loaded in MNs was analyzed by HPLC. An array of NLC-MNs was dissolved in $7.0 \mathrm{~mL}$ of $2 \%$ Tween 80 in distilled water for 1 hour under moderate stirring (300 rpm). The solution was diluted with methanol and sonicated for 5 minutes to dissolve the NR from the NLC-MNs. The distribution behavior of NR in NLC-MNs was determined by fluorescence microscopy and confocal laser scanning microscopy (CLSM) as described below. A z-series was rotated (x: $72.5^{\circ}, y: 316.7^{\circ}, \mathrm{z}: 287.7^{\circ}$ ) and stacked perpendicularly.

\section{Determination of skin penetration and distribution}

Distribution of NR in the skin was determined using a Franz diffusion cell, as described above, with the membrane filter replaced by excised back skin from a 15-month-old minipig. The skin was sectioned in 600- to $700-\mu \mathrm{m}$ slices using a microdermatome (B Braun Melsungen AG, Melsungen, Germany) and cut into $3 \times 3 \mathrm{~cm}$ pieces. The skins were wrapped in aluminum foil and stored at $-20^{\circ} \mathrm{C}$. Prior to the experiment, the frozen skin was pre-equilibrated in phosphate buffer saline to thaw completely. Hydrated skin was then placed at the flat bottom and wiped with a tissue to remove liquid. The MN array was inserted into the center of the skin $\left(1.76 \mathrm{~cm}^{2}\right)$ with a force of $20.0 \mathrm{~N}$ for 1 minute using a weight device. The skin with MNs was then carefully mounted in the Franz diffusion cell. Other processes were carried out as described above.

After the 24-hour MN array application period, NR in the receptor compartment was detected using HPLC, and the skin sample was removed and separated from the MN array, which was then assayed for NR by HPLC. The specimen was positioned parallel to the bottom of the mold, surrounded with Tissue-Tek ${ }^{\circledR}$ optimum cutting temperature compound 4583 (Sakura Finetek USA, Inc., Torrance, CA, USA), and flash frozen in liquid nitrogen. The frozen tissue was sectioned from the SC to the bottom using a cryotome (Ultracut UCT; Leica Microsystems, Wetzlar, Germany) at $-25^{\circ} \mathrm{C}$. Slice thickness was set at $50 \mu \mathrm{m}$ and four consecutive slices were taken and placed into the same glass vial. This procedure was repeated until the entire section was sliced. ${ }^{15}$ To each vial, $2.5 \mathrm{~mL}$ of methanol was added and sonicated for 1 hour at $40^{\circ} \mathrm{C}$. This step was repeated twice. The extracted NR was quantified by HPLC assay.

\section{Fluorescence microscopy}

An inverted fluorescence microscope (Motic AE31; Speed Fair Co, Ltd) with 480-nm excitation and 535-nm emission filters (Motic MHG-100B; Speed Fair Co, Ltd) was used to investigate the entrapment of NR in NLC-MNs and the distribution of NR in minipig skin. Digital images were captured using a camera (Moticam Pro 825A; Speed Fair Co, Ltd) controlled with Motic Image Advanced 3.2 software.

\section{CLSM}

CLSM (LSM 510; Carl Zeiss Meditec AG, Jena, Germany) was performed to scan optical sections at different z-positions along the optical axis (z-sectioning). NR was excited at $543 \mathrm{~nm}$ and its emission was detected with a longpass filter (LP 560; Carl Zeiss). The NLC-MNs were placed on a microscope slide and scanned by CLSM to visualize the distribution of fluorescent NR-containing nanocarriers. Images were acquired in a series of the xy-sections (parallel to the base plate of MNs) as successive focal planes along the z-axis. Scanning was conducted at an interval of $56 \mu \mathrm{m}$ 
from the tip of the MN through the z-axis, vertical to the xy-plane.

\section{Visualization of skin distribution behavior}

The skin sample was acquired as described above. A scalpel was used to excise the tissue exposed to the MN array. This tissue was positioned perpendicularly to the bottom of the mold and frozen with optimum cutting temperature compound in liquid nitrogen. The frozen skin was sectioned vertically using a cryotome and the $30-\mu \mathrm{m}$ sections were investigated using light and fluorescence microscopy, as described above. The images from the two techniques were merged to assess the distribution of NR in the different skin layers.

To observe the distribution pattern of NR in a timedependent manner, skins treated with NLC-MNs were removed from Franz diffusion cells at time points of 1, 4, 8, 12 , and 24 hours. The skins were separated from MN arrays and observed using CLSM.

Scanning was conducted at intervals of $60 \mu \mathrm{m}$ from the SC. Images were taken automatically until a red channel response was detected. All other CLSM measurement settings (except scanning interval) were the same as those described above. For further comparison of CLSM images, pixel analyses were performed using the Adobe Photoshop ${ }^{\circledR}$ histogram feature (Adobe Systems Incorporated, San Jose, CA, USA). According to the intensity of redness, ranging from 0 (black) to 255 (red), four regions were categorized: invisible (less than 64); mild (64 to 127); moderate (128 to 191); and strong (more than 192). The number of pixels representing redness in every region were counted and expressed as the redness occupancy of the total 4,624 microphotograph pixels.

\section{Statistical analysis}

All reported data are mean \pm standard deviation. The differences between groups were analyzed by Student's $t$-test and considered to be statistically significant where $P<0.05$.

\section{Results}

\section{HPLC NR assay validation}

The seven-point calibration curve was linear in the concentration range of $5-100 \mathrm{ng} / \mathrm{mL}$ NR $(\mathrm{y}=6680.984 \mathrm{x}+1615.498$, $\left.r^{2}=0.9999\right)$. The calibration standards for intraday and interday accuracy and precision are listed in Table 1. Intraday and interday precision ranged from $1.31 \%$ to $3.74 \%$ and from $2.10 \%$ to $2.56 \%$, respectively. The maximum values for intraday and interday variation at $10 \mathrm{ng} / \mathrm{mL}$ were 1.779 and 4.815 , respectively.
Table I Intraday and interday accuracy and precision of the nile red assay

\begin{tabular}{lrll}
\hline $\begin{array}{l}\text { Spiked } \\
\text { concentration } \\
(\mathbf{n g} / \mathbf{m L})\end{array}$ & $\begin{array}{l}\text { Detected } \\
\text { concentration } \\
(\mathbf{n g} / \mathbf{m L})\end{array}$ & $\begin{array}{l}\text { Precision } \\
\text { (RSD) }\end{array}$ & $\begin{array}{l}\text { Accuracy } \\
\text { (\% DEV) }\end{array}$ \\
\hline $\begin{array}{l}\text { Intraday variation } \\
10\end{array}$ & $9.651 \pm 0.361$ & 3.740 & 1.779 \\
60 & $60.969 \pm 1.416$ & 2.322 & -1.400 \\
100 & $100.809 \pm 1.316$ & 1.306 & -0.792 \\
Interday variation & $9.518 \pm 0.199$ & 2.094 & 4.815 \\
10 & $61.365 \pm 1.573$ & 2.563 & -2.275 \\
60 & $100.272 \pm 2.468$ & 2.461 & -0.272 \\
\hline 100 &
\end{tabular}

Notes: RSD $=($ standard deviation $/$ mean $) \times 100 . \% \mathrm{DEV}=($ spiked concentration measured concentration) $/$ spiked concentration $\times 100$.

Abbreviations: RSD, relative standard deviation; \% DEV, percentage deviation.

\section{Characteristics of NLCs}

NR-loaded NLCs were successfully prepared using the HPH technique and the size, polydispersity index, zeta potential, and entrapment of NR are listed in Table 2. The particle size was in the range of 250-295 nm with polydispersity index values of approximately 0.273 . Zeta potential was approximately $-20 \mathrm{mV}$. Entrapment of NR into NLCs was evaluated in terms of drug loading and EE. A high load of NR was entrapped into NLCs, with the EE being greater than $99 \%$.

\section{In vitro release profile of NR from NLCs}

The in vitro release of NR from NLCs is shown in Figure 2A. The NLC dispersion showed a controlled release of NR for 24 hours, and the release reached plateau at around 36 hours. Over $75 \%$ of the NR in the NLC dispersion was released within 24 hours. In comparison, a micellar solution composed of $5 \%$ Tween 80 released NR more rapidly than the NLC dispersion and reached a plateau at around 9 hours, which indicated that $96 \%$ of the NR was released.

\section{Characterization of NLC-MNs}

The specifications of the MNs were as follows: length, $350 \mu \mathrm{m}$; base diameter, $380 \mu \mathrm{m}$; and dip diameter, $30 \mu \mathrm{m}$.

Table 2 Physicochemical properties of NR-loaded NLCs

\begin{tabular}{ll}
\hline Physical characteristics & \\
Z-average size $(\mathrm{nm})$ & $268.4 \pm 16.2$ \\
PDI & $0.273 \pm 0.02 \mathrm{I}$ \\
Zeta potential $(\mathrm{mV})$ & $-20.4 \pm 0.6$ \\
Drug entrapment & $1.025^{*}$ \\
NR loading (mg NR/g lipid) & $100^{*}$ \\
\hline NR encapsulation efficiency $(\%)$ &
\end{tabular}

Note: *NR was not detected in the ultrafiltrate (LOD was found to be $0.01 \mathrm{ng} / \mathrm{mL}$ ). Abbreviations: LOD, limit of detection; NLCs, nanostructured lipid carriers; NR, nile red; PDI, polydispersity index. 

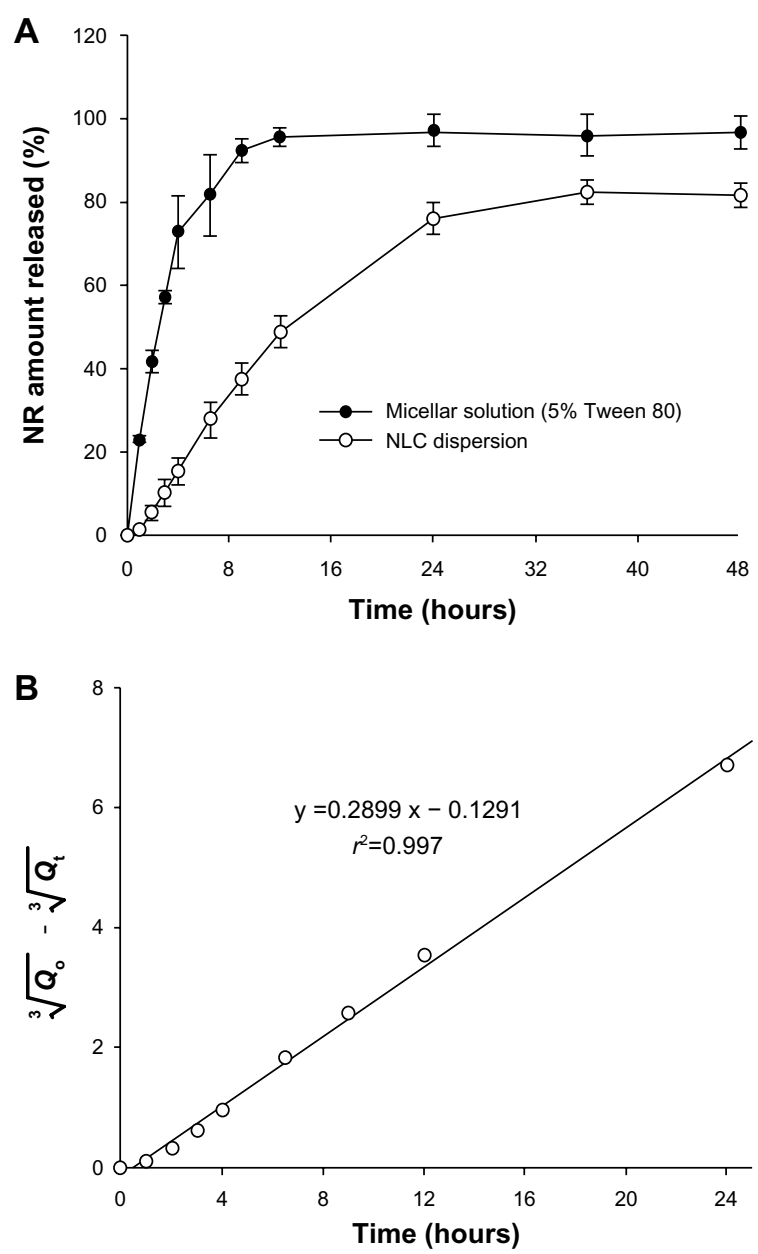

Figure 2 In vitro release profiles of NR from NLC dispersion and micellar solution (A) and curve fitting to the Hixson-Crowell equation for NLC dispersion for 24 hours (B).

Note: Data represent mean \pm standard deviation $(n=3)$.

Abbreviations: NLC, nanostructured-lipid-carrier; NR, nile red.

Standard deviations were below $10 \%$ of each value, except those of tip diameter. The aspect ratio, defined as the ratio of length to base diameter, was approximately 0.9 (Table 3). This value was lower than those of other common conical-shaped polymeric MNs, which generally ranged from 1.2 to 5 , suggesting that these MNs were short in length.

Table 3 Physicochemical properties of the NLC-MNs

\begin{tabular}{lc}
\hline Physical characteristics $(\mathrm{n}=2 \mathrm{I})$ & \\
Length $(\mu \mathrm{m})$ & $347.80 \pm 15.26$ \\
Base diameter $(\mu \mathrm{m})$ & $385.47 \pm 30.13$ \\
Tip diameter $(\mu \mathrm{m})$ & $29.45 \pm 6.97$ \\
Aspect ratio & $0.91 \pm 0.08$ \\
Drug entrapment $(\mathrm{n}=3)$ & \\
NR loading (ng/array) & $189.8 \mathrm{I} \pm 12.13$ \\
\hline
\end{tabular}

Note: Values are the mean \pm standard deviation.

Abbreviations: NLC-MNs, nanostructured-lipid-carrier-loaded microneedles; NR, nile red.

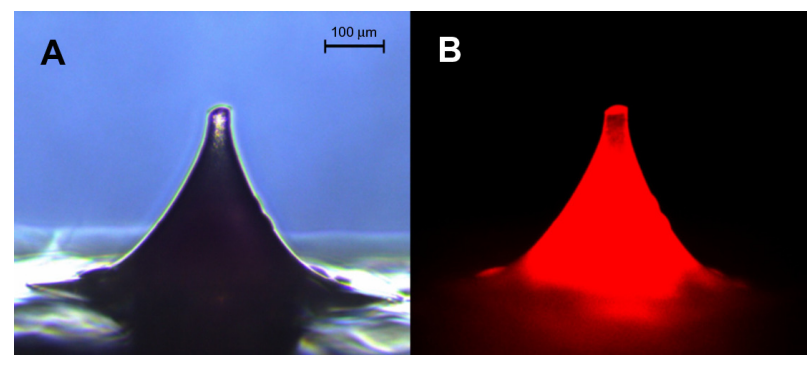

Figure 3 Microscopic images of NLC-MNs under normal (A) and fluorescent (B) light sources.

Abbreviation: NLC-MNs, nanostructured-lipid-carrier-loaded microneedles.

Fluorescence microscopic imaging of NLC-MNs proved that NLCs were distributed evenly throughout the MNs, as shown in Figure 3. This finding was also supported by CLSM analysis (Figure 4). Even distribution of the nanocarrier was found in fluorescence images in the xy-plane (horizontal view) and z-series (perpendicular direction). In the xy-plane micrographs arranged in z-series tiers, fluorescence was well distributed, but there were gaps in the center of the bottom portion (approximately 224-392 $\mu \mathrm{m}$ from the tip). For further visualization, the micrograph images were rotated and stacked together to analyze the distribution of fluorescence and the shape of the NLC-MNs.

\section{Quantitative analysis of skin localization of NR}

This experiment measured relative NR distribution (Table 4). Approximately $70 \%$ of the NR dosage was distributed in the skin, of which $40 \%$ was localized in the upper layer of the skin $(0-200 \mu \mathrm{m})$. The residual amount of NR in the NLC-MNs was limited to $10 \%$, and the cumulative amount of NR that permeated into the receptor fluid was approximately $5 \%$. There was a loss less than $15 \%$ of the NR in mass balance.

Table 4 NR localization after 24-hour NLC-MN array application on minipig skin in a Franz diffusion cell

\begin{tabular}{lc}
\hline Recovery site & Amount of NR (\%)* \\
\hline Residual in NLC-MN & $10.44 \pm 5.46$ \\
$0-200 \mu \mathrm{m}$ & $38.97 \pm 3.66$ \\
$200-400 \mu \mathrm{m}$ & $22.23 \pm 1.95$ \\
Over $400 \mu \mathrm{m}$ & $8.28 \pm 4.5 \mathrm{I}$ \\
Receptor compartment & $5.67 \pm 1.71$ \\
Total & $85.63 \pm 7.17$ \\
\hline
\end{tabular}

Notes: Values are the mean \pm standard deviation $(n=3)$. *Percent of applied amount of NR (189.9 ng).

Abbreviations: NLC-MN, nanostructured-lipid-carrier-loaded microneedle; NR, nile red. 


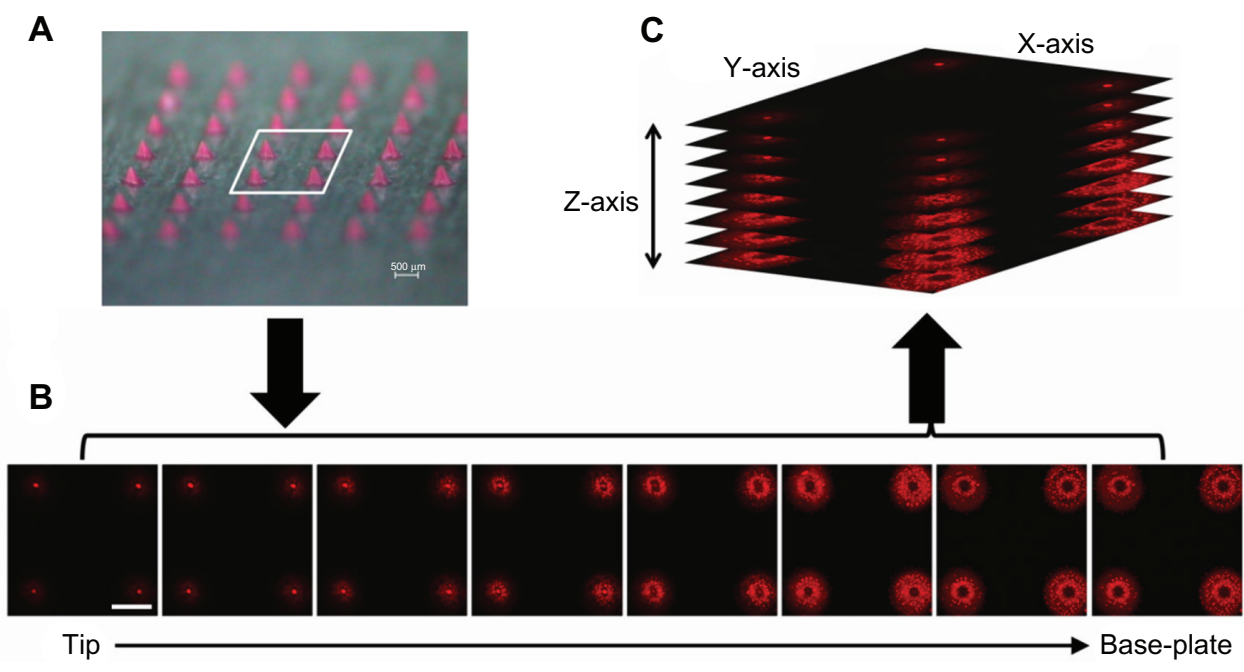

Figure 4 CLSM images of NR-NLC distribution in the MNs revealing even distribution of the nanocarrier throughout the needle.

Notes: (A) Microscopic image of NLC-MN array. (B) Confocal images of the xy-plane in z-series (sequential xy sections, interval $=56 \mu \mathrm{m})$. $(\mathbf{C}) \mathrm{z}$-series rotated $\left(\mathrm{x}\right.$ : $72.5^{\circ}$, y: $316.7^{\circ}, \mathrm{z:} 287.7^{\circ}$ ) and stacked perpendicularly. The white scale bar represents $500 \mu \mathrm{m}$.

Abbreviations: CLSM, confocal laser scanning microscopy; NLC-MN, nanostructured-lipid-carrier-loaded microneedle; NR, nile red; MNs, microneedles.

\section{Visualization of skin distribution behavior}

Figure 5 depicts fluorescent and light microscopy images of vertical skin sections following application of NLC-MNs for 24 hours. Strong fluorescent intensity was observed in the epidermis, while it was more diffuse throughout the dermal layer.

Images of the skin scanned by CLSM at different depths (z-series) showed that the fluorescence appeared to spread out radially with increased intensity as time passed (Figure 6). After the application of NLC-MNs for 1 hour, fluorescence was detected in the spots pierced by MNs. Between 4 hours and 8 hours, the fluorescence permeated into the vicinity of the pierced spots. After 12 hours, CLSM detected fluorescence in deeper skin layers, throughout the deep dermal tissues.

\section{Discussion}

The advantages of using MNs for delivering various active molecules to deeper dermal tissues and/or the systemic

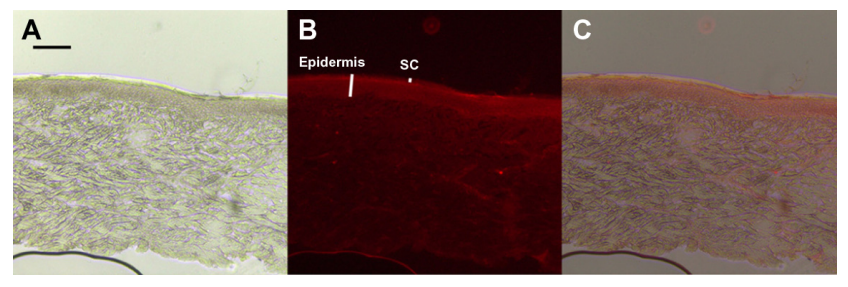

Figure 5 Microscopic images of minipig skin after NLC-MN application for 24 hours (A) under normal light, (B) under fluorescent light, and (C) a merged image of (A) and (B).

Notes: The red color in (B) and (C) represents NR. The black scale bar represents $100 \mu \mathrm{m}$.

Abbreviations: NLC-MN, nanostructured-lipid-carrier-loaded microneedle; NR, nile red; SC, stratum corneum. circulation have been described previously., ${ }^{3,11,27,28}$ Puncturing the SC allows efficient dermal/transdermal delivery of not only small compounds such as calcein, vitamin $C$, and ketoprofen, but also of large molecules including vaccines, gene vectors, and antibodies. In the case of lipophilic drugs, permeation is not a matter of concern for dermal delivery, because of their easier partitioning into the SC than that of other drugs. Many lipophilic drugs are therefore commercially available as creams and/or ointments. ${ }^{29-31}$ Nevertheless, controlled delivery and localization of the drug to dermal layers are still necessary. MNs can deliver the drug with high efficiency in a controlled or sustained release pattern.

The selection of polymers, the matrix body architecture, and the means of drug loading are principal factors for $\mathrm{MN}$ technology. In addition, microparticulate carriers encapsulating a drug could be combined with MNs for successful drug delivery. The release of a hydrophilic probe by using microparticle-loaded MNs has been investigated, indicating that the release rate of calcein could be controlled by manipulation of the properties of the hydrogel particles. ${ }^{32}$ PLGA nanoparticle-loaded polymeric MNs have also been studied as vehicles to enhance the dermal delivery and localization of a hydrophobic molecule. This PLGA-MN system exhibited improved delivery of NR to the skin, with $3.59 \%$ of the total NR in the MNs being localized to the skin, compared to $0.13 \%$ from a control patch. ${ }^{15}$ Another PLGA-MN system facilitated controlled antigen delivery to skin dendritic cells. A single immunization with this system was sufficient to induce an antiviral adaptive immune response and to acquire protective immunity against a respiratory virus. ${ }^{17}$ Puncture of 
A 1 hour

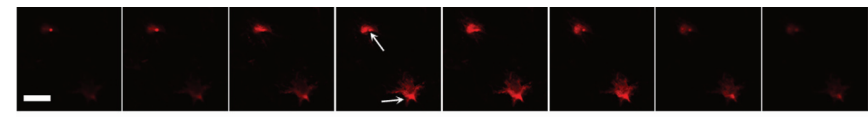

B 4 hours

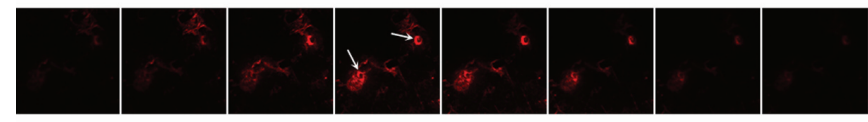

C 8 hours

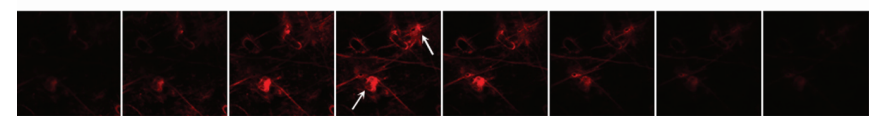

D 12 hours

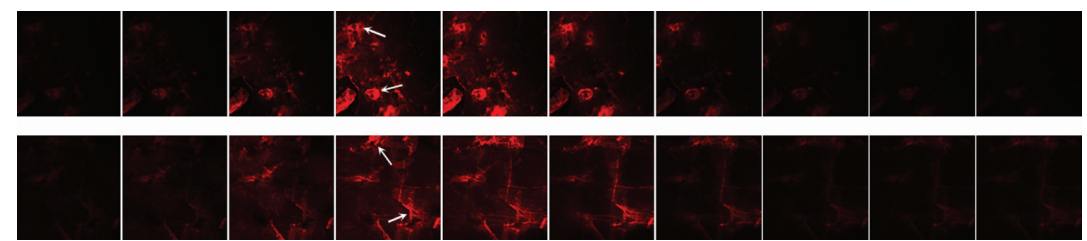

Figure 6 CLSM images of a z-series (sequential xy sections, interval =60 $\mu \mathrm{m}$ ) after application of NLC-MNs to minipig skin for (A) I hour, (B) 4 hours, (C) 8 hours, (D) 12 hours, and (E) 24 hours.

Notes: The arrows indicate the spot pierced with the MNs. The white scale bar represents $500 \mu \mathrm{m}$.

Abbreviations: CLSM, confocal laser scanning microscopy; NLC-MNs, nanostructured-lipid-carrier-loaded microneedles; MNs, microneedles.

skin is crucial for drugs with narrow therapeutic windows to avoid over- or under-dosing, and incorporation of the drug into a carrier can maintain the level within a therapeutic range by controlling drug release. ${ }^{12}$

In the present study, NLCs were designed as a lipid-based nanocarrier for controlled delivery of NR, a hydrophobic model probe, and engrafted into a hydrophilic HA-based MN. Since NLCs are composed of liquid oil and solid lipid, oil-soluble drugs are easily entrapped with high EE. Our results showed perfect encapsulation of NR (Table 2) because of its very poor solubility in aqueous media. ${ }^{33}$ The kinetics of NR release from the NLC dispersion for the period of 24 hours were calculated using the Hixson-Crowell equation as follows: $Q_{o}^{1 / 3}-Q_{t}^{1 / 3}=K_{\mathrm{HC}} \times t$, where $Q_{o}$ was the initial amount of drug in the NLC, $Q_{t}$ was the remaining amount of drug in the NLC at time $t$, and $K_{\mathrm{HC}}$ was a constant incorporating the surface-volume relationship. As shown in Figure 2B, this model fitted the release of NR in the present study $\left(r^{2}=0.997\right)$. It described the NR release by dissolution and bioerosion, accompanied by changes in surface area and diameter of the NLC, suggesting complete biodegradation of the nanocarrier after releasing its contents. Meanwhile, the release profile of NR from the nanoemulsion was similar to that from the NLC (data not shown). This showed that sustained release of NR from the NLC was most strongly influenced by the oil component, rather than the solid lipid phase, of the carrier.

Nanocarriers, including NLCs, do not easily reach the deeper layers of the skin. ${ }^{34}$ This means that delivery of low amounts of drug would not allow deeper tissues to be targeted. ${ }^{35}$ However, micropuncture of the skin using
MNs enables drug delivery to deep dermal layers. ${ }^{15}$ The aspect ratio, expressed as the relative value of length to base diameter, is one of the crucial parameters determining the insertion capacity of MNs through the skin. The NLCMNs used in this study were rather short, since the aspect ratio was much lower than those of other common conical polymeric MNs. The lower the aspect ratio of the $\mathrm{MN}$, the harder it is for it to pierce the skin. ${ }^{11}$ Nevertheless, as shown in Figure 6, the delivery of NR was successfully achieved with our NLC-MN application. Strong fluorescence of NR in the dermal layer indicated the efficient localization effect of the system. This finding was corroborated by quantitation, demonstrating that approximately $70 \%$ of the NR was localized in the skin. Moreover, the fluorescence spread out radially to a larger area of the deep dermis as time passed. This time-dependent distribution behavior was further visualized by regional pixel analyses based on the intensity of redness. Figure 7 illustrates the three-dimensional cumulative distribution behavior of the NR by the variables of both time of application and depth of dermal layer. Moderate and strong redness regions peaked at 12 to 24 hours and in the 240 to $360 \mu \mathrm{m}$ range. When the mild redness region was included, the redness occupancy at the same range was tripled. These results were consistent with previous literature describing the usefulness of nanoparticles with $\mathrm{MNs}$ for increasing skin penetration of drug and its deposition in the upper layer of the skin. ${ }^{15,16}$

The enhanced delivery efficiency of the NLC-MNs could be explained in two stages, as illustrated by step-wise controlled dermal delivery of NR (Figure 8). Firstly, MNs perforate the SC and liberate the NLCs as the polymeric matrix 

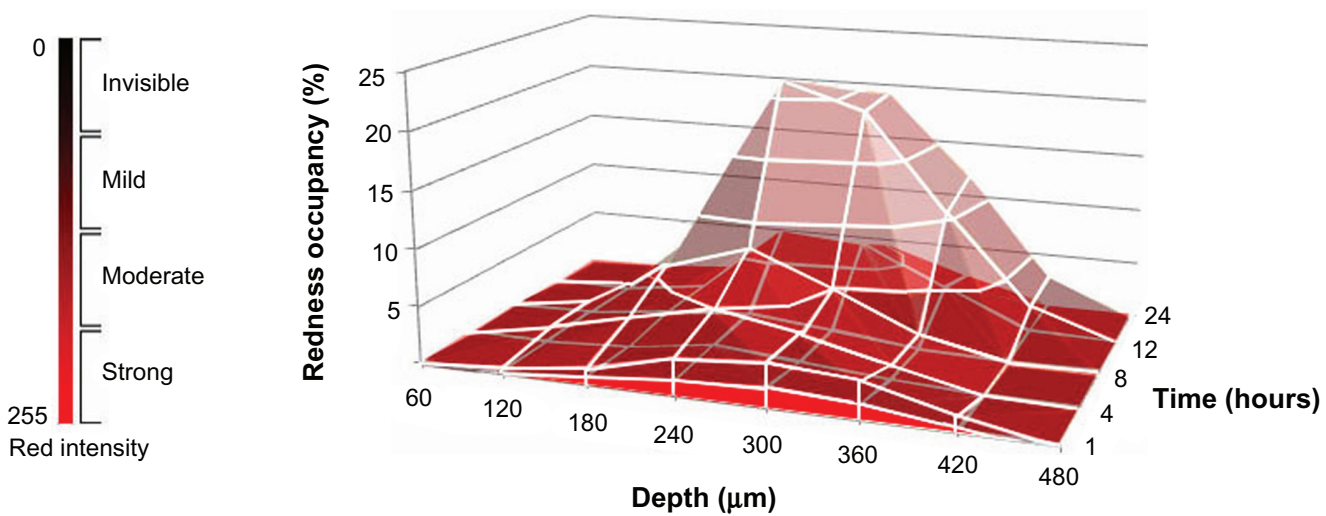

Figure 7 Three-dimensional cumulative distributional behavior of NR, based on pixel analyses of CLSM images after application of NLC-MNs to minipig skin.

Notes: The redness occupancy is represented by two different panels: bottom panel for regions with strong and moderate redness; overlaid panel for the mild redness region.

Abbreviations: CLSM, confocal laser scanning microscopy; NLC-MNs, nanostructured-lipid-carrier-loaded microneedles; NR, nile red.

A

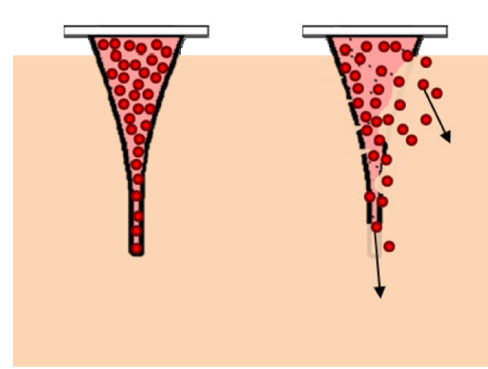

B

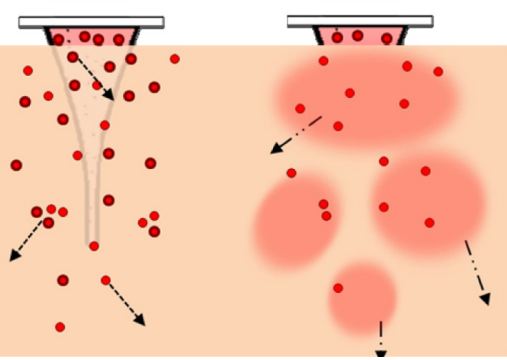

Figure 8 Step-wise illustration of the controlled dermal delivery of a lipophilic drug by NLC-MNs.

Notes: (A) Immediately after the application of the NLC-MNs to the skin. (B) Bioerosion of the MNs to release the NLCs carrying the drug. (C) Liberated NLCs diffuse and/or partition into the dermal layer while releasing the drug. (D) Drug is totally exposed and orientates to the target sites in dermal tissue. Solid black or grey lines represent the surface of the MNs as intact or eroded, respectively. Red colored circles represent the NLCs containing NR (as a model lipophilic molecule).

Abbreviations: NLC-MNs, nanostructured-lipid-carrier-loaded microneedles; NR, nile red; MNs, microneedles; NLCs, nanostructured-lipid-carriers.

erodes. Since HA is a biocompatible and bioerodible polymer, the matrix body rapidly melts down and delivers the loaded nanoparticles. Numerous studies using bioerodible HA-based MNs have been carried out. Biodegradable HA-MNs fabricated with an appropriate geometry and sufficient strength for insertion into the skin dramatically increased transdermal transport of molecules. ${ }^{14}$ HA-MNs rapidly dissolved in the skin's extracellular fluid after application. ${ }^{6,14}$ Secondly, NR was released from the liberated NLCs in a controlled manner and finally reached the target site. During this stage, the NLCs diffused and/or partitioned into the specific dermal layer while releasing the drug slowly. As discussed earlier, NR release from the NLCs was controlled by both diffusion and surface erosion, according to Hixson-Crowell's cube root law. The NLCs then decomposed to their elementary components, which would be digested by endogenous lipases, followed by biochemical dissipation of the resulting fatty acid molecules.
In summary, the NLC-MNs developed in this study might provide a useful platform for developing a sophisticated dermal delivery system for lipophilic compounds. However, further studies on their optimization and practical application for therapeutic drug delivery are still necessary.

\section{Conclusion}

NLCs containing NR, a model lipophilic compound, were successfully incorporated into hydrophilic polymeric MNs. A skin permeation study using a Franz diffusion cell with minipig dorsal skin confirmed the efficient localization of NR to the upper layer of the skin. The present study also demonstrated that dermal delivery of NR could be controlled by NLC-MNs.

\section{Acknowledgment}

This study was supported by the Seoul R and BD program (SS100001). 


\section{Disclosure}

The authors report no conflicts of interest in this work.

\section{References}

1. Wiechers JW. The barrier function of the skin in relation to percutaneous absorption of drugs. Pharm Weekbl Sci. 1989;11(6):185-198.

2. Gill HS, Denson DD, Burris BA, Prausnitz MR. Effect of microneedle design on pain in human volunteers. Clin J Pain. 2008;24(7): $585-594$.

3. Henry S, McAllister DV, Allen MG, Prausnitz MR. Microfabricated microneedles: a novel approach to transdermal drug delivery. J Pharm Sci. 1998;87(8):922-925.

4. Donnelly RF, Singh TR, Woolfson AD. Microneedle-based drug delivery systems: microfabrication, drug delivery, and safety. Drug Deliv. 2010;17(4):187-207.

5. Jin CY, Han MH, Lee SS, Choi YH. Mass producible and biocompatible microneedle patch and functional verification of its usefulness for transdermal drug delivery. Biomed Microdevices. 2009;11(6): $1195-1203$.

6. Katsumi H, Liu S, Tanaka Y, et al. Development of a novel selfdissolving microneedle array of alendronate, a nitrogen-containing bisphosphonate: evaluation of transdermal absorption, safety, and pharmacological effects after application in rats. J Pharm Sci. 2012;101(9):3230-3238.

7. Donnelly RF, Morrow DI, Singh TR, et al. Processing difficulties and instability of carbohydrate microneedle arrays. Drug Dev Ind Pharm. 2009;35(10):1242-1254.

8. Gittard SD, Ovsianikov A, Monteiro-Riviere NA, et al. Fabrication of polymer microneedles using a two-photon polymerization and micromolding process. J Diabetes Sci Technol. 2009;3(2): 304-311.

9. Ito Y, Yoshimitsu J, Shiroyama K, Sugioka N, Takada K. Self-dissolving microneedles for the percutaneous absorption of EPO in mice. J Drug Target. 2006;14(5):255-261.

10. Lee $\mathrm{K}$, Lee $\mathrm{CY}$, Jung H. Dissolving microneedles for transdermal drug administration prepared by stepwise controlled drawing of maltose. Biomaterials. 2011;32(11):3134-3140.

11. Park JH, Allen MG, Prausnitz MR. Biodegradable polymer microneedles: fabrication, mechanics and transdermal drug delivery. $J$ Control Release. 2005;104(1):51-66.

12. Park JH, Allen MG, Prausnitz MR. Polymer microneedles for controlled-release drug delivery. Pharm Res. 2006;23(5): $1008-1019$.

13. Kogan G, Soltés L, Stern R, Gemeiner P. Hyaluronic acid: a natural biopolymer with a broad range of biomedical and industrial applications. Biotechnol Lett. 2007;29(1):17-25.

14. Liu S, Jin MN, Quan YS, et al. The development and characteristics of novel microneedle arrays fabricated from hyaluronic acid and their application in the transdermal delivery of insulin. $J$ Control Release. 2012;161(3):933-941.

15. Donnelly RF, Morrow DI, Fay F, et al. Microneedle-mediated intradermal nanoparticle delivery: Potential for enhanced local administration of hydrophobic pre-formed photosensitisers. Photodiagnosis Photodyn Ther. 2010;7(4):222-231.

16. Zhang W, Gao J, Zhu Q, et al. Penetration and distribution of PLGA nanoparticles in the human skin treated with microneedles. Int J Pharm. 2010;402(1-2):205-212.

17. Zaric M, Lyubomska O, Touzelet $\mathrm{O}$, et al. Dendritic cell targeting via microneedle arrays laden with antigen-encapsulated poly-d,l-lactideco-glycolide nanoparticles induces efficient antitumor and antiviral immune responses. ACS Nano. 2013;7(3):2042-2055.
18. Müller RH, Radtke M, Wissing SA. Solid lipid nanoparticles (SLN) and nanostructured lipid carriers (NLC) in cosmetic and dermatological preparations. Adv Drug Deliv Rev. 2002;54 Suppl 1:S131-S155.

19. Puglia C, Blasi P, Rizza L, et al. Lipid nanoparticles for prolonged topical delivery: An in vitro and in vivo investigation. Int J Pharm. 2008:357(1-2):295-304.

20. Angelov B, Angelova A, Papahadjopoulos-Sternberg B, Hoffmann SV, Nicolas V, Lesieur S. Protein-containing PEGylated cubosomic particles: Freeze-Fracture Electron Microscopy and Synchrotron Radiation Circular Dichroism Study. J Phys Chem B. 2012;116(26):7676-7686.

21. Angelova A, Angelov B, Drechsler M, Garamus VM, Lesieur S. Protein entrapment in PEGylated lipid nanoparticles. Int J Pharm. 2013;454(2): 625-632.

22. Angelov B, Angelova A, Filippov SK, et al. DNA/fusogenic lipid nanocarrier assembly: millisecond structural dynamics. J Phys Chem Lett. 2013;4(11):1959-1964.

23. Lee K, Jung H. Drawing lithography for microneedles: A review of fundamentals and biomedical applications. Biomaterials. 2012;33(30): 7309-7326.

24. Zhang X, Pan W, Gan L, Zhu C, Gan Y, Nie S. Preparation of a dispersible PEGylate nanostructured lipid carriers (NLC) loaded with 10-hydroxycamptothecin by spray-drying. Chem Pharm Bull (Tokyo). 2008;56(12):1645-1650.

25. Lee SG, Jeong JH, Kim SR, et al. Topical formulation of retinyl retinoate employing nanostructured lipid carriers. J Pharm Invest. 2012;42(5): 243-250.

26. Teeranachaideekul V, Müller RH, Junvaprasert VB. Encapsulation of ascorbyl palmitate in nanostructured lipid carriers (NLC) - Effects of formulation parameters on physicochemical stability. Int $J$ Pharm. 2007;340(1-2):198-206.

27. Oh JH, Park HH, Do KY, et al. Influence of the delivery systems using a microneedle array on the permeation of a hydrophilic molecule, calcein. Eur J Pharm Biopharm. 2008;69(3):1040-1045.

28. Noh YW, Kim TH, Baek JS, et al. In vitro characterization of the invasiveness of polymer microneedle against skin. Int J Pharm. 2010;397(1-2):201-205.

29. Pardeike J, Schwabe K, Müller RH. Influence of nanostructured lipid carriers (NLC) on the physical properties of the Cutanova Nanorepair Q10 cream and the in vivo skin hydration effect. Int J Pharm. 2010;396(1-2):166-173.

30. Mrowietz U, Wustlich S, Hoexter G, Graeber M, Bräutigam M, Luger T. An experimental ointment formulation of pimecrolimus is effective in psoriasis without occlusion. Acta Derm Venereol. 2003;83(5): 351-353.

31. Godfrey HR, inventor; Godfrey HR, assignee. Topical zinc compositions and methods of use. United States patent US 6558710. May 6, 2003.

32. Kim M, Jung B, Park JH. Hydrogel swelling as a trigger to release biodegradable polymer microneedles in skin. Biomaterials. 2012;33(2): 668-678.

33. Greenspan P, Fowler SD. Spectrofluorometric studies of the lipid probe, nile red. J Lipid Res. 1985;26(7):781-789.

34. Lombardi Borgia S, Regehly M, Sivaramakrishnan R, et al. Lipid nanoparticles for skin penetration enhancement-correlation to drug localization within the particle matrix as determined by fluorescence and parelectric spectroscopy. J Control Release. 2005;110(1): 151-163.

35. Cevc G, Vierl U. Nanotechnology and the transdermal route: A state of the art review and critical appraisal. J Control Release. 2010;141(3): 277-299. 
International Journal of Nanomedicine

Dovepress

\section{Publish your work in this journal}

The International Journal of Nanomedicine is an international, peerreviewed journal focusing on the application of nanotechnology in diagnostics, therapeutics, and drug delivery systems throughout the biomedical field. This journal is indexed on PubMed Central, MedLine, CAS, SciSearch ${ }^{\circledR}$, Current Contents ${ }^{\circledR} /$ Clinical Medicine,
Journal Citation Reports/Science Edition, EMBase, Scopus and the Elsevier Bibliographic databases. The manuscript management system is completely online and includes a very quick and fair peer-review system, which is all easy to use. Visit http://www.dovepress.com/ testimonials.php to read real quotes from published authors.

Submit your manuscript here: http://www.dovepress.com/international-journal-of-nanomedicine-journal 